\title{
An Investigation on the Barriers and Facilitators of the Implementation of Electronic Health Records (EHR)
}

\author{
Fereshteh Farzianpour*, Sara Amirian*, Raziye Byravan \\ Department of Health Management and Economics, School of Public Health, \\ Tehran University of Medical Sciences, Tehran, Iran \\ Email: farzianp@sina.tums, *amirian_s2@yahoo.com
}

Received 10 September 2015; accepted 12 December 2015; published 15 December 2015

Copyright (C) 2015 by authors and Scientific Research Publishing Inc.

This work is licensed under the Creative Commons Attribution International License (CC BY). http://creativecommons.org/licenses/by/4.0/

(c) (i) Open Access

\section{Abstract}

The application of technology in health care, in the form of electronic health records (EHR), is the most important and necessary issue in order to improve the quality of health care, and studies have shown that, not only is it a way to integrate information and represent the condition of patients, and a dynamic source for health care, however it leads to gain access to clinical information and records, electronic communications, comprehensive training and management, and ultimately enhancing the public health; the aim of this study is to investigate the factors influencing the implementation of EHR, which are known as barriers and facilitators. The research is conducted in the form of a review research, and with the help of the Keywords of EHR; barriers and facilitators, articles, from 2008 to 2013, were searched and studied in the Internet-databases. The results of the studies show that the most effective factors include: efficiency, motivation, management, and the participation of end users. Factors such as technical aspects ease of use, available resources, and human resources, have limited effects. And security and privacy, the expected output, lack of time, and workload have relative effects, and also the relation between the patient and clinical staff, has no effects in the process of implementing EHR.

\section{Keywords}

Electronic Health Records (EHR), Barriers and Facilitators

\section{Introduction}

Information technology has had a profound impact on various businesses; the health care institutions have not

${ }^{*}$ Corresponding authors.

How to cite this paper: Farzianpour, F., Amirian, S. and Byravan, R. (2015) An Investigation on the Barriers and Facilitators of the Implementation of Electronic Health Records (EHR). Health, 7, 1665-1670.

http://dx.doi.org/10.4236/health.2015.712180 
been an exception either. E-health is a new arena between information, public health, and commerce [1]. The application of technology in health care, in the form of EHR, is the most important and necessary issue in order to improve the quality of health care, and studies have shown that, not only is it a way to integrate information and represent the condition of patients, and a dynamic source for health care, however it leads to gain access to clinical information and records, electronic communications, comprehensive training and management, and ultimately, enhancing the public health [2]. The priority of countries for the establishment of e-health is different, and its constituents are referred to by different names, e-health is founded based on EHR [3].

EHR is a confidential and secure record related to an individual's whole lifetime that includes information about the individual's health care records in the health care system. This record is electronically accessible for authorized service providers at any time and any places, and it is designed in order to facilitate data sharing between health care providing organizations [4]. This definition is presented by the World Health Organization [5].

In a study in Canada, it has been shown that EHR is a key factor for the integration of different systems, which makes a safe and effective health care possible for every Canadian [6] that can improve the quality of care for patients, through sharing relevant, timely, and updated information among a care providing team. [4]

The resistance of physicians and other health care staff towards the adoption and use of health information systems and electronic medical records is one of the important barriers that delays the successful adoption and implementation of such systems [7]. The Medical Institute comments that the broad adoption of EHR can play a pivotal role in improving the patient safety and health care quality, and also it may reduce the costs of providing outpatient services [8].

And also several studies identify facilitators and barriers to the adoption of EHR, such as: costs, difficult implementation, resistance of physicians, and organizational features, such as: the hospital size, ownership, and training status [9].

In addition to the above mentioned cases, resistance of some medical and professional staff towards changing from a manual system to an electronic system may be a problem in developing countries. Most health and information managers are aware that this change may take time or moderate the behavior and motivation of health staff to a small extent. The reason for changing from a manual system to an electronic system is important. The attitude toward EHR is not constant, and this is both a challenge and strength in different societies [10]. For this reason, the physicians' active support, and use of EHR for its benefits are required, and identifying the possible barriers to the implementation, from the physicians' view, is essential and important [11].

In a study, conducted on the successful selection and implementation of EHR in small outpatient institutions in the United States of America, it is shown that the experience of the implementation of EHR depends on various factors that include: technology, training, leadership, the change management process, and the unique characteristics of the outpatient environment [5].

But despite the potential benefits of EHR, its implementation is facing executive limitations and barriers, the most important limitations include: cost limitations, technical limitations, standardization limitations, individuals' attitudinal-behavioral limitations, and organizational limitations, researches show that individuals' attitudinal-behavioral limitations or resistance to change plays a greater role than other limitations [12].

In general, extensive attention has been paid to the capacity of EHR for reducing the health-care costs and significant improvement of the health care quality [13]. Despite these attentions and serious concerns about the implementation of EHR in developed and developing countries, studies have shown that there is a wide gap between planning to start e-health systems and success in the implementation of such systems, especially in developing countries, as well as achieving the primary objectives and expected benefits [7] [14], that indicates the existence of serious barriers on the way of the implementation of this project, and it requires attention to the facilitators of the program.

Hence, this study tries to identify the barriers to and facilitators of the implementation of EHR and provide solutions to overcome the barriers and take advantage of the facilitators in order to optimize the operation of implementing this important issue.

\section{Method}

The research method is a review research. The keywords of EHR; barriers to and facilitators of EHR were searched in these databases: SID, ELSEVIER, and PUBMED. 
Several criteria were intended for searching the articles that include the following items:

1) The year of publication: The articles related to the years before 2008 were excluded;

2) Articles, whose full texts can be downloaded for free, were investigated;

3) Articles which contained the barriers and facilitators were extracted;

4) Articles about the EHR subsystems were excluded.

Finally, 19 articles were selected according to the above criteria, and their full texts were studied Figure 1.

\section{Findings}

In a study conducted by Marie-Pier Gagnon in Canada, 10 factors influencing the implementation of EHR are mentioned. These 10 factors include: perceived usefulness, efficiency, motivation, participation of end-users in the implementation, the interaction between the patient and clinical staff, lack of time and workload, available resources, management, the expected output, and interdepartmental interaction capability.

Another study entitled "Barriers to the adoption of Electronic Health Records" has expressed that one of the barriers is denial and resistance by physicians, which is for a variety of reasons, such as: Lack of time, high costs, lack of computer skills, disruption in the working procedure, concerns about security and privacy, disruption in communication among users, disruption in physician-patient relationship, lack of motivation, complexity, lack of adequate physical space, concerns about the inability to choose a suitable EHR system, lack of technical supports, lack of interdepartmental interaction capability, the lack of access to computers and computer literacy, lack of trust in sellers, concerns about entering data in the system, insufficient training by sellers after selling the system and lack of physicians' access to the sellers' technical supports, insufficient exchange of data, concerns about the acceptance of the system by patients, inadequate formal training and training classes, low speed in some units, lack of the system integrity for inpatients and outpatients, and lack of wireless communication with some nursing homes and clinics [8].

The results of a study in Saudi Arabia, divided the barriers to the implementation of EHR, into 6 categories: barriers related to human resources, financial barriers, legal barriers, organizational barriers, technical barriers, barriers related to hospital staff [7]. Also, in another categorization, barriers and facilitators were classified into 6 categories:

1) Features related to users: Learning, typing skills, perceived usefulness, motivation, strategy (procedure), and other items such as: Remembering or forgetting passwords, and how to work with the system;

2) Features related to the system: Hardware or software features, speed, system performance, and usability;

3) Supports from other groups: Technical supports, formal training, informal supports from colleagues

4) Organizational supports: Extra time (working with the system or communicating with patients), and intraorganizational integration

5) Environmental factors: Physical space, wireless communication, and social environment (a physician does

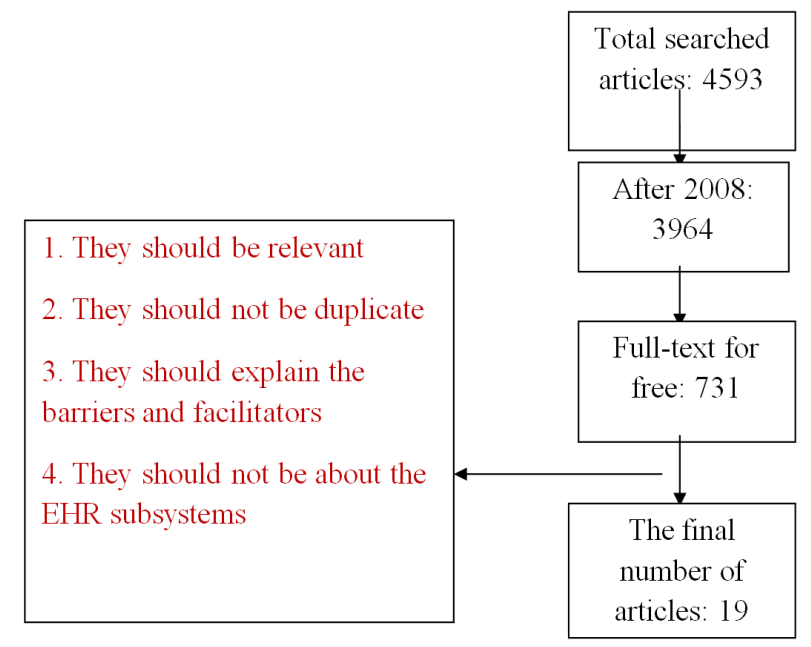

Figure 1. Related to the searched articles. 
not feel comfortable, when typing and talking to the patient at the same time);

6) General controls: Compulsory use of EHR and the lack of any alternative such as a print or copy facilitate the use of the system, but in cases where no type of data were entered about a patient, this would be considered a barrier [9].

A study by Mokhtari et al. in Isfahan, has investigated the challenges and barriers to the implementation of EHR, from physicians', administrators' and intellectuals' point of view, and classified them into two groups: infrastructural and structural, so that the challenges related to the infrastructure include 4 categories: issues related to information technology, lack of a common language between designers and users and lack of uniform definitions and contents, cultural issues, and lack of needs assessment. And the structural challenges include 3 categories: Instability in the implementation, legal violations, lack of integration and sharing the investment [10].

In Canada, 10 important factors were identified as the barriers to and facilitators of the implementation of EHR, from the viewpoint of the users (managers, physicians, patients and hospital staff), that include: Concerns regarding the design and technical aspects of the project, ease of use, interdepartmental performance, concerns about privacy and security of data, the issue of cost, efficiency, ability and familiarity with the system, motivation, interaction between patient and health care staff, lack of time and workload [4] [5]. Another division has dealt with these aspects as barriers:

A. Financial: The high start-up cost, the cost of maintaining, managing and controlling the system, uncertain payback period, lack of financial resources (budget).

B. Technical: Lack of computer skills, lack of technical supports and trainings, system complexity, system limitations, lack of adapting to the users' needs, lack of trust, the need for standardization, lacking in hardware.

C. Time: The time required for selecting, buying, and implementing the system, the time required for learning the system, the time required for entering the data, further time for each patient, the time required for transfer data from paper documents to computer.

D. Psychological: Lack of trust in the system, the need for control.

E. Social: Lack of trust in sellers, lack of support from other local and national organizations, intervention in the physician-patient relationship, lack of support from other colleagues, lack of support from managerial levels

F. Legal: Concerns about security and privacy of data.

G. Organizational: The size of organization, and the type of organization.

$\mathrm{H}$. The process of change: Lack of support from organizational culture, lack of motivation, lack of participation, lack of leadership [11].

The executive barriers to the implementation of EHR in Uremia were identified and classified in this way: Technical limitations, standardization, organizational changes, individuals' attitudinal and behavioral barriers, and costs [12]. From another view, the barriers are divided into two categories: Personal: disruption in the working procedure, lack of understanding of the benefits, privacy and security of data, usability and flexibility, lack of time for training and redesigning the work processes, lack of computer skills, and organizational: financial costs, lack of adequate informational resources, problems of implementation, designing and testing the software, and the facilitators of the implementation include: the users' motivation, saving the physicians' time, choosing the appropriate type of system, the provability of usefulness, adequate IT systems [15].

From another aspect, the barriers and facilitators can be expressed as follows:

Barriers: The density of the data, information transfer, abnormal signals, being a multitasking system, ergonomics, IT architecture, documentation load, workload, training, and required skills;

Facilitators: Efficiency and availability, readiness, connection between experts, preference of providers, completeness of records, detecting errors, educational tools, and establishing a relation between the patient and provider, adaption to the users' needs, and management of working processes and procedures [16]. Also financial, ethical, legal, social, and technical barriers, and facilitators such as: financial supports from the government and insurance companies, and participation of staff were identified in another study [17].

\section{Discussion}

The results of the studies reviewed, show that the two important factors; the participation of end users in selecting and planning, and the status of the physicians' salaries, are considered as the main barrier to the adoption of EHR by physicians. Also, understanding the factors facilitating the implementation plays a key role in the success of the system. The two factors; motivation and the perceived usefulness of the project from the users' point 
of view, should be taken into consideration in the time of implementation. These two factors are closely related to each other, inasmuch as the perceived usefulness of the system increases the motivation for using it [4].

The findings show that there are a lot of barriers to the implementation of EHR, among which individuals' attitudinal and behavioral limitations and organizational changes, have obtained a high score [12]. According to the factors mentioned in the study, improving the ability of experts for the easy and effective use of the system, enhances the quality and improves the safety, through the use of EHR [8]. The results of the studies showed that the most effective factors include: efficiency, motivation, management, and the participation of end users. And factors such as technical aspects ease of use, available resources, and human resources, have limited effects. And security and privacy, the expected output, lack of time, and workload have relative effects, and also the relation between the patient and clinical staff, has no effects, in the process of implementing EHR [13] [18].

In this regard, the identification of barriers, such as: data, information, infrastructure, data exchange standards, and processing of codes and the vocabulary list of these systems, is of particular importance. Due to the challenges and complexities of EHR, the creation of this system requires a purposeful strategy, because it imposes changes to the way of performing the tasks that requires compatibility and adaptability to clinical processes. Therefore, the creation of these systems makes necessary the cooperation of different groups, such as: providers, users, designers, and experts in health information management (medical records) [19] [20].

\section{Conclusions}

EHR is a complicated and multidimensional project, that many factors will affect its implementation. A factor, in a situation, can be as a barrier, yet it can also be considered as a facilitator, such as motivation. If users do not have the sufficient motivation to work with the system, they will resist towards it and will refuse to perform it, while in another situation and condition, if the necessary motivation is provided, it will contribute to the project as a facilitator. What is certain is that cost and time are always regarded as two deterrent factors.

For a successful implementation of the project, it is necessary to evaluate the readiness of the organization for the adoption and implementation. Individuals' attitudinal and behavioral barriers such as resistance to change and pessimism about the future of the project need changes in the culture and atmosphere of the organization. Also since EHR is an extensive and large-scale project, it needs intersect oral coordination and requires basic infrastructures, such as telecommunication infrastructures, and message exchange standards, and the government support and private sector investment, to compensate the costs associated with start-up, maintenance, and control of the project.

\section{Providing Suggestions and Solutions}

Describing the benefits of EHR for the public.

Advertising through mass media.

Providing incentives for people to accept it.

Identifying the users' perspective.

Development and improvement through training.

Identifying and defining the informational needs of patients, providers, and other clients.

Holding coordination meetings with the managers of relevant organizations before the implementation of HER.

\section{Limitation}

In this research we had restriction on the language for many articles. Data collections were articles Farsi and English.

\section{References}

[1] Renner, A.L. and Swart, J.C. (1997) Patient Core Data Set. Standard for a Longitudinal Health/Medical Record. Computers Informatics Nursing, 15, S7-S13.

[2] International Standards for Business, Government and Society (2006) http://www.iso.org/iso/search.htm?qt=ISO+18308\&searchSubmit=Search\&sort=rel\&type=simple\&published=on/

[3] Simborg, D.W. (2008) Promoting Electronic Health Record Adoption. Is It the Correct Focus? Journal of the American 
Medical Informatics Association, 15, 127-129. http://dx.doi.org/10.1197/jamia.M2573

[4] Seied Farajollah, S. (2007) Structure Minimum Data Set in Electronic Health Record Presentation. Proceeding of the 2nd Regional Congress about Medical Record, Shiraz, 2007, 23-24.

[5] Gagnon, N., Shaw, N. and Sicotte, C. (2009) Users' Perspectives of Barriers and Facilitators to Implementing EHR in Canada: A Study Protocol. Implementation Science, 4, 20. http://dx.doi.org/10.1186/1748-5908-4-20

[6] Torabi, M. and Safdari, R. (2004) Electronic Health Record. Behineh Publication, Tehran.

[7] International Standards Worldwide (1996) http://www.astm.org/index.shtml/

[8] International Standards for Business, Government and Society. http://www.iso.org/iso/page_not_found.htm?errurl=/isotc/catalogue.detail.htm\&querystring=csnumber=33397

[9] Holden, R.J. and Saf, P. (2011) What Stands in the Way of Technology-Mediated Patient Safety Improvements? A Study of Facilitators and Barriers to Physicians' Use of Electronic Health Records. Journal of Patient Safety, 7, 193203. http://dx.doi.org/10.1097/PTS.0b013e3182388cfa

[10] Mokhtari, H., Jahanbakhsh, M. and Tavakoli, N. (2011) Challenges of EHR Implementation and Related Guidelines in Isfahan. Procedia Computer Science, 3, 1199-1204. www.sciencedirect.com

[11] Boonstra, A. and Broekhuis, M. (2010) Barriers to the Acceptance of Electronic Medical Records by Physicians from Systematic Review to Taxonomy and Interventions. BMC Health Services Research, 10, 231. http://www.biomedcentral.com/1472-6963/10/231 http://dx.doi.org/10.1186/1472-6963-10-231

[12] Cotea, C. (2010) Research Coordination Unit, CMVH. Electronic Health Record Adoption: Perceived Barriers and Facilitators Mayne Medical School Herston Road, Herston QLD 4006.

[13] Barriers and Facilitators to Using the HER. https://www.google.com/url?sa=t\&rct=j\&q=\&esrc=s\&source=web\&cd=6\&cad=rja\&ved=0CFIQFjAF\&url

[14] Reza, M. and Razavi, H. (2013) Electronic Health Records Acceptance and Implementation in Developing Countries: Challenges and Barriers. Razavi International Journal of Medicine, 1, 11-6. http://dx.doi.org/10.5812/rijm.15077

[15] (2004) Document Summary Copyright 2005 ASTM International C585-90. www.shurfitproducts.com/astm-c585.pdf/

[16] Gunter, T.D. and Terry, N.P. (2005) The Emergence of National Electronic Health Record Architectures in the United States and Australia: Models, Costs, and Questions. Journal of Medical Internet Research, 7, e3. http://dx.doi.org/10.2196/jmir.7.1.e3

[17] (2007) HL7 EHR System Functional Model: A Major Development towards Consensus on Electronic Health Record System Functionality. http://www.sanita.forumpa.it/documenti/0/100/140/148/EHR-SWhitePaper.pdf/

[18] (2005) Open Clinical: Standards: CDA—Clinical Document Architectur. www.openclinical.org/std_cda.htm/

[19] Ferranti, J.M., Musser, R.C., Kawamoto, K. and Hammond, W.E. (2006) The Clinical Document Architecture and the Continuity of Care Record: A Critical Analysis. Journal of the American Medical Informatics Association, 13, 245252. http://dx.doi.org/10.1197/jamia.M1963

[20] (2009) ISO/TC 215 from Wikipedia, the Free Encyclopedia. http://en.wikipedia.org/wiki/ISO/TC_215/ 